\title{
Editorial. Suspicion et exception
}

\section{Emmanuel-Pierre Guittet et Miriam Perier}

\section{(2) OpenEdition}

1 Journals

\section{Édition électronique}

URL : http://journals.openedition.org/conflits/1822

DOI : 10.4000/conflits.1822

ISSN : $1777-5345$

Éditeur :

CCLS - Centre d'études sur les conflits lilberté et sécurité, L'Harmattan

\section{Édition imprimée}

Date de publication : 1 juin 2005

Pagination : 5-12

ISBN : 2-7475-8911-0

ISSN : 1157-996X

\section{Référence électronique}

Emmanuel-Pierre Guittet et Miriam Perier, «Editorial. Suspicion et exception », Cultures \& Conflits [En ligne], 58 | été 2005, mis en ligne le 10 octobre 2005, consulté le 30 mars 2021. URL : http:// journals.openedition.org/conflits/1822; DOI : https://doi.org/10.4000/conflits.1822

Ce document a été généré automatiquement le 30 mars 2021.

Creative Commons License 


\title{
Editorial. Suspicion et exception
}

\author{
Emmanuel-Pierre Guittet et Miriam Perier
}

« La peur n'a jamais été favorable à la liberté. Or notre temps et notre société sont caractérisés par

le souci d'une plus grande sécurité, sous toutes

ses formes. Lorsque sécurité et liberté sont susceptibles d'entrer en conflit, ce n'est pas la liberté qui l'emporte dans ce choix $»^{1}$.

1 Ce nouveau numéro de Cultures \& Conflits sur "suspicion et exception » poursuit la réflexion engagée dans nos publications précédentes sur les dispositifs, les logiques et les stratégies antiterroristes à l'œuvre en Europe et aux Etats-Unis. Les contributions présentées arguent de la nécessité de se départir des discours qui reconstruisent chaque attentat comme la naissance, sans cesse renouvelée, d'un nouvel âge du terrorisme. Comme dans les numéros 44 et $56^{2}$, on s'écarte ici des pratiques, des réflexions et de ces discours qui font des attentats et des politiques antiterroristes engagées à leur suite des "réactions » à la violence, et des «innovations » politicotechniques imaginatives. Sans négliger la répétition d'une violence politique de grande ampleur et l'interrogation sur son caractère de menace existentielle, il nous semble que les prétendues nouvelles réponses sont en fait de bien vieilles solutions. Le recours à tous les moyens offerts par la technologie - l'usage accru et l'échange des bases de données de surveillance, de la biométrie, de la transnationalisation des données des services de renseignement et de la police -, comme le postulat de la nécessité d'une coopération accrue entre les services de police et de renseignement, au-delà du national et dans un esprit «naturel » de concorde pour faire face précisément à un danger "nouveau ", étaient déjà présents dans la panoplie des justifications des discours fondateurs de la CIPC, l'ancêtre d'Interpol et on promettait que le marconigramme et l'anthropométrie juguleraient l'anarchisme. On ne peut raisonnablement considérer le 11 septembre comme la marque d'un nouvel âge de l'antiterrorisme sans participer à ces discours de peur et à ces justifications des technologies de surveillance et de contrôle du moment. En revanche, analyser comment l'angoisse du futur et une volonté de vengeance sont les éléments innovants des politiques antiterroristes post 11 septembre nous engage à saisir comment ces 
politiques antiterroristes du «bord du monde », de la "pré-apocalypse », de l'exception ont changé durablement les relations de pouvoir, plaçant d'une part les Etats-Unis dans une position de force - à la fois au niveau international et au sein des débats internes de l'Union européenne -, et favorisant d'autre part le retour à des mesures anciennes, coercitives et proactives, qui diminuent l'importance de la sauvegarde en termes de vie privée, de protection de données et d'habeas corpus. Si nouveauté il y a, alors elle serait plutôt dans la routinisation d'une violence et du cycle provocation/répression à une échelle transnationale, dans la convergence de pratiques dérogatoires déjà éprouvées, dans un discours de surenchère à la peur que l'on cherche à gérer en inquiétant et en réassurant tour à tour et, finalement, dans la généralisation de technologies de contrôle des personnes en mouvement et de surveillance de ceux profilés comme indésirables ou dangereux. Pratiques d'exception et logique de suspicion : l'objet de ce numéro est d'abord une analyse de ce passage de la figure du coupable, qui suppose que l'action a déjà eu lieu, à la figure du suspect où l'action n'est pas encore. Et c'est moins le 11 septembre 2001 qui est au cœur de ce numéro, que ce problème du glissement dans la temporalité du traitement des pratiques de la violence.

2 En présentant dans ce numéro la recherche initiée par trois des équipes du programme-cadre de recherche ELISE ${ }^{3}$, ce numéro offre d'autres perspectives d'analyse afin de comprendre ces discours de peur et engage dans une toute autre réflexion sur les rapports entre les pratiques d'exception, les logiques d'inimitié et de suspicion à l'œuvre aujourd'hui. En déplaçant le curseur de l'analyse des attentats et de la recherche d'une explication immédiate vers une compréhension des politiques antiterroristes et des rapports entre pratiques d'exception et logiques d'inimitié et de suspicion, les chercheurs engagés dans le programme ELISE entendent apporter un éclairage renouvelé et résolument critique de ces "réponses" politiques qui conduisent certains à donner pour neuf ce qui ne l'est pas, et qui amènent d'autres, voire les mêmes, sous couvert d'une conception extensive de l'urgence, à faire de la suspicion une « certitude » et de cette certitude une preuve de la culpabilité.

Dans ce numéro, la recherche conduite dans le cadre du programme européen ELISE montre comment ces politiques ne résolvent toutefois pas le problème actuel de l'utilisation possible par des groupes clandestins d'une violence disruptive, mais contribuent au contraire à son aggravation, en simplifiant le monde en catégories binaires, en faisant appel au raisonnement du scénario du pire, en créant un climat de suspicion détruisant ainsi toute cohésion sociale au nom d'un certain patriotisme, et en attisant les sentiments d'injustice au niveau local, national et mondial. Faut-il rappeler que ces politiques, à défaut d'être véritablement efficaces, ont avant tout été le produit de circonstances particulières, du comportement de certains professionnels de la politique et des relations entretenues par ces derniers avec les professionnels de la sécurité et du renseignement? Elles offrirent aussi des circonstances favorables aux intérêts propres de certains acteurs et, de la sorte, à la transformation d'une situation en mouvement, avec de nombreuses opportunités, en un «fait accompli». Ainsi, par exemple, le choix d'envahir l'Afghanistan et l'Irak « en réponse à Al Qaeda ", l'option prise de la voie militaire plutôt que celle d'une approche judiciaire et policière - comme les autorités espagnoles l'ont fait après les attentats de Madrid du 11 mars 2004 -, ont ouvert la voie à une vision américaine unilatérale. Selon cette vision le reste du monde n'est que le backyard des Etats-Unis, et ces derniers ont autant la force que le droit d'exiger obéissance à leurs choix stratégiques, à leur logique clanique du « avec nous ou 
contre nous $»^{4}$. Dans cette logique, la guerre devient la solution d'évidence à ces formes de violence transnationales, même si les ennemis sont secrets, cachés, clandestins ou plus exactement « furtifs ». En ce sens, Rob Walker, discutant des limites d'une analyse en termes d'international, d'impérial et d'exception schmittienne (ou benjaminienne), montre que la guerre peut être interprétée comme un fragment d'une restructuration beaucoup plus vaste de la capacité à décider des exceptions. Elle n'est pas la disparition de la souveraineté, mais bien la multiplication et la prolifération des souverainetés, de souverainetés ne ressemblant que peu à ce que nous associons d'ordinaire aux Etats modernes.

4 Ce retour à des doctrines de sécurité nationale élargies à l'ensemble du monde se nourrit des logiques d'exception où la sécurité devient la valeur clé, délaissant ainsi la liberté et la justice à d'autres temps ou les reconfigurant en tant qu'auxiliaires de la sécurité. Force est de constater que cette sécurité devient une valeur unifiée et mondialisée dans les discours, tandis que la liberté se voit fragmentée et divisée en nombreux « morceaux » cruellement restreints par les dérogations s'imposant au nom de la nécessité du moment, et de la croyance de certains hommes politiques que leur rôle est d'agir coûte que coûte, quoi qu'il arrive. La sécurité n'a-t-elle plus de frontières? Elle engendre au moins un discours de la nécessité à une coopération renforcée. Cette dernière est ainsi devenue la pierre angulaire d'une information visant à anticiper les événements, distinguant qui est dangereux de qui ne l'est pas, qui est suspect de qui ne l'est pas, qui a le droit d'entrer de qui ne l'a pas. Les différents accords de coopération (formels ou informels) au sein de l'Union européenne d'une part, et avec les Etats-Unis d'autre part, sont allés dans ce sens, participant ainsi de la (re)structuration de l'identité de l'UE: des démocraties luttant «naturellement " contre les mêmes ennemis, les terroristes. La prétention au succès de ces politiques découle d'un raisonnement spécifique portant sur le futur, sur l'éventualité d'un " Armageddon » et sur un raisonnement en termes de "scénario du pire » (worst case scenario) dans lequel ce n'est pas la probabilité qu'un risque se produise qui est calculée sinon le fait qu'il est envisageable qu'il survienne mais alors avec des dommages tels sur la population qu'il doit être anticipé à tout prix. Cette position ontologique du "scénario du pire» a un vaste héritage historique d'éléments structurant des politiques sécuritaires, en particulier les politiques sécuritaires américaines, comme le montre Didier Bigo. Cela change fondamentalement la définition du calcul rationnel car celui-ci est fondé sur une volonté de maîtriser le futur et ses virtualités. Mais ce souci de détermination du futur par anticipation s'appuie sur une scientifisation de l'astrologie relabellisée "renseignement» et "prévention", par le biais de la surveillance, de la mise en registre des données biométriques des individus ainsi que par l'établissement de profils types (profiling). Penser que rassurer les populations passe par l'adoption systématique de nouvelles mesures antiterroristes ne consiste-t-il pas à jouer avec les peurs prises pour être celles de tout un chacun? Un sentiment de réconfort plus ou moins fallacieux suffit-il à justifier et légitimer des pratiques de plus en plus illibérales d'Etats dits libéraux? Il ne faut pas minorer l'impact de ces arguments dans la mesure où ils jouent de la croyance de certains que des institutions supérieures et secrètes sont forcément détentrices de la vérité et du savoir sur le futur, dans un moment où le retour des certitudes cherche à échapper aux contraintes du monde sublunaire par évocation d'un dieu ou d'un futur anticipable.

5 Le succès de ces discours en faveur de la réintroduction d'un état d'esprit guerrier au sein du pays ainsi que la justification de la diminution des libertés publiques au nom de 
l'urgence n'est pas sans lien avec les luttes entre professionnels de la sécurité et de la politique au niveau transnational. Cela a créé une ambivalence sur le statut de la guerre comparé aux luttes internes et aux conflits de classe au sein de la société. La thématique de l'ennemi de l'intérieur, ou plus exactement de l'ennemi «infiltré » est au coeur des priorités des services de renseignement et, depuis les attentats du 11 septembre 2001, au coeur des priorités des professionnels de la politique. Sommes-nous en guerre au sein de nos sociétés? Quel est le véritable statut de "l'urgence nationale »? En analysant les réactions des services de renseignement, Laurent Bonelli souligne combien chaque bureaucratie a sa propre réponse, logiquement liée à son histoire, à ses intérêts premiers et à ses normes. La police et les juges refusent en majorité la lecture de la menace en termes de guerre et considèrent que le terrorisme est d'abord un crime, voire un crime contre l'Humanité, mais pas une guerre au sein de la société. En revanche, les services de renseignement, et les partisans de la guerre asymétrique cherchent à imposer cette idée d'un terrorisme apocalyptique notamment par le biais des armes de destruction massive ou encore en invoquant un «Pearl Harbor informatique »-, menace véritable à la survie, pour donner des raisons au renforcement des politiques de l'exception, justifiée par la nouveauté de la menace terroriste.

$6 \quad$ Et pourtant, les dispositifs antiterroristes actuels ont une histoire très directement liée aux années 1970 et poursuivent plus ou moins des structures identiques, en y ajoutant toutefois autant d'innovations technologiques que possible. La volonté de se réapproprier la maîtrise des frontières, d'introduire de nouvelles technologies permettant de tracer l'individu, de renforcer les filtres contre ceux qui souhaitent entrer, d'emprisonner les suspects potentiels, d'utiliser l'armée et les services de renseignement pour «faire la police " à l'intérieur du pays, de se faire l'avocat de la nécessité de mesures dérogatoires au nom d'un futur cataclysmique ont toutes déjà été utilisées et ont toutes déjà été refoulées, dans les années 1970, par l'émergence de droits civiques en faveur des citoyens et des étrangers ainsi que par l'amélioration de la protection des données. Comme le montre Philippe Bonditti, la tension entre ce retour à des méthodes coercitives anciennes de contrôle et de surveillance - nourri des fantasmes du tout technologique et de ce rêve politique d'une gestion possible de nos devenirs par anticipation -, débouche sur des pratiques de contrôle et de surveillance faisant de la traçabilité des individus la condition de sécurité des espaces et des populations, et de l'échange d'information en temps réels entre acteurs de la sécurité, la condition de réussite de ces pratiques.

7 Or, le débat sur la meilleure politique de sécurité commune fondée sur une meilleure coopération est trompeur s'il n'est pas corrélé à l'impact sur les libertés civiques de la vision commune de la sécurité, en particulier lorsque cela tend à devenir la mondialisation de la surveillance des personnes en mouvement. L'indispensable débat sur la démocratie et sur la liberté, que nous sommes effectivement en train de sacrifier au nom de libertés futures et de l'éradication de la menace terroriste, n'est toujours pas à l'ordre du jour malgré des tentatives par trop éparses et souvent discréditées par avance. Même si la peur du terrorisme, notamment dans ses liens prétendus avec les armes de destruction massive, était moins importante, elle a réarticulé le continuum de l'insécurité créé au milieu des années 1980 au nom de la lutte contre la libre circulation des individus et la peur d'une "invasion" de l'Occident par des migrants. Comme l'analyse très justement Valsamis Mitzilegas, cette peur irrationnelle a induit une certaine disproportionalité des réponses administratives, policières et judiciaires 
européennes, relatives à l'accès, à la circulation et à la protection des données, ainsi qu'un contournement des instances démocratiques dans la mise en place de ces politiques. Les discours ont connecté le terrorisme, l'immigration, et les pratiques de l'Islam entre les deux continents via des logiques de suspicion.

8 Les politiques de l'exception et du danger permanent, justifiant l'étendue de mesures d'exception au-delà de zones, de personnes et de temporalités limitées ont renouvelé avec une conception très souverainiste et très traditionnelle du politique -, le droit de l'autorité politique à dire l'exception, là où la raison d'Etat n'a pas à être justifiée ni à supporter les droits de l'Homme et encore moins le jus cogens. L'histoire contemporaine nous apprend combien les gouvernements des Etats libéraux ont toujours fait valoir la protection du citoyen pour restreindre les libertés et justifier leur droit de déclarer un état d'urgence, un état d'exception partiel ou général, disposant ainsi d'un chèque en blanc auquel les juges doivent se référer tant que les limites de l'exception ont été validées par le Parlement. Ne faut-il pas " défendre la société » ? C'est du moins au nom de cette vision que se développe une forme de prétention unilatérale et exclusive de l'exécutif à dire l'exception. Dans son article, Elspeth Guild examine ce nouveau cadre de la liberté et de la sécurité, qui a créé des tensions entre la classe politique et les juges, en portant son attention sur l'élément crucial de la détention indéfinie, de l'Etat de droit et du rôle des juges face aux pouvoirs exécutifs du gouvernement et de l'administration. L'Etat de droit a été invoqué comme principe capable de compenser un faible contrôle démocratique sur les processus exécutifs et législatifs dans la protection des droits à la liberté individuelle. Les libertés et la cohésion sociale sont liées - d'un point de vue juridique - à plusieurs facteurs : le contrôle des systèmes de pouvoir complexes et divers, les moyens politiques et administratifs à l'implémentation de dispositions légales sur des questions de discriminations entre autres.

9 Nous ne pouvons pas prétendre que nous vivons dans un monde en perdition, et qu'il s'agirait dès lors de tenter d'équilibrer la liberté et la sécurité car ce discours, à la limite de la démocratie, représente un moyen et une justification propres à favoriser la sécurité de certains en diminuant ou en retirant la liberté et certains droits fondamentaux à d'autres. La pensée démocratique a toujours eu à se battre contre ce genre d'évidences trompeuses. Ces politiques et leurs justifications se trompent en ce qu'elles minimisent les valeurs qu'elles disent vouloir défendre. Ces réactions de peur, de colère, de vengeance ont pour effet de créer un unanimisme (tout comme la volonté de trouver un bouc émissaire), posant les conditions au développement d'une rhétorique de "guerre » avec une sorte de régression à l'habitus de la mentalité de la Guerre Froide, si ce n'est celle du maccarthisme ou encore celle des années 1930 : celle d'un ennemi à traquer au nom de la paradoxale sauvegarde des principes fondamentaux de la démocratie. La démocratie est un processus jamais accompli et toujours à sauvegarder. Cet enjeu ne doit-il pas passer par des mesures immédiates pour réunifier la liberté et refragmenter la sécurité ? Cette réunification ne passe-t-elle pas, dès lors, par une mise en commun d'acteurs significatifs au sein de l'Union européenne, par le renforcement de leur pouvoir et la mise en place d'outils de protection adéquats des droits fondamentaux, contre les nouvelles sources d'action discrétionnaire, et en particulier les réseaux de contrôle au niveau européen? 


\section{NOTES}

1.. Errera R., Les libertés à l'abandon, Paris, Seuil, 1975, p. 298.

2.. « Défense et identités. Un contexte sécuritaire global ? », Cultures \& Conflits, Paris, L'Harmattan, n44, hiver 2001 ; « Militaires et sécurité intérieure. L'Irlande du Nord comme métaphore ", Cultures \& Conflits, Paris, L'Harmattan, n56, hiver 2004.

3.. ELISE, European Liberty and Security, est un programme de recherche de type PCRD ( $5^{\text {ème }}$ génération) financé par la Commission européenne. Il regroupe depuis octobre 2002 sept universités européennes dont : l'Université de Nijmegen (Pays-Bas), l'Université de Keele (Royaume-Uni), et l'Institut d'Etudes Politiques de Paris (France) dont nous publions ici les contributions. Voir http://www.eliseconsortium.org. 4.. Nous reviendrons plus amplement dans un prochain numéro de la revue sur les implications de la logique militaire de la guerre au terrorisme et sur le statut de la guerre dans notre monde contemporain. Ce prochain numéro sera l'occasion de publier une seconde série de recherches conduites dans le cadre du programme ELISE par d'autres partenaires européens, dont l'équipe italienne de l'Université de Gênes et l'équipe anglaise du King's College.

\section{INDEX}

Mots-clés : exception

\section{AUTEURS}

\section{EMMANUEL-PIERRE GUITTET}

Miriam PERIER est titulaire d'un DEA de sociologie politique (Université Paris-I) et chargée de recherches au centre d'études sur les conflits.

\section{MIRIAM PERIER}

Miriam PERIER est titulaire d'un DEA de sociologie politique (Université Paris-I) et chargée de recherches au centre d'études sur les conflits. 